\title{
Recent developments and on-line tests of uranium carbide targets for production of nuclides far from stability
}

V.N. Panteleev ${ }^{\mathrm{a}}$, O. Alyakrinskiy ${ }^{\mathrm{b}}$, A. Andrighetto ${ }^{\mathrm{b}}$, A.E. Barzakh ${ }^{\mathrm{a}}$, M. Dubois ${ }^{\mathrm{c}}$, C. Eleon ${ }^{\mathrm{c}}, \mathrm{S}$. Essabaa $^{\mathrm{d}}$, O. Bajeat ${ }^{\mathrm{d}}$, D.V. Fedorov ${ }^{\mathrm{a}}$, G. Gaubert ${ }^{\mathrm{c}}$, A.M. Ionan ${ }^{\mathrm{a}}$, V.S. Ivanov ${ }^{\mathrm{a}}$, P. Jardin ${ }^{\mathrm{c}}$, A. Lanchais $^{b}$, C. Lau ${ }^{d}$, R. Leroy ${ }^{c}$, G. Lhersonneau ${ }^{b}$, C. Mhamed ${ }^{d}$, K.A. Mezilev ${ }^{\mathrm{a}}$, P.L. Molkanov ${ }^{\mathrm{a}}$, F.V. Moroz ${ }^{a}$, S.Yu. Orlov, ${ }^{a}$ V. Rizzi ${ }^{b}$, M.G. Saint Laurent ${ }^{c}$, L. Stroe ${ }^{b}$ L.B. Tecchio ${ }^{b}$, A.C.C. Villari $^{\mathrm{c}}$, Yu.M. Volkov ${ }^{\mathrm{a}}$

a) 188350, Petersburg Nuclear Physics Institute RAS, Gatchina, Leningrad district, Russia

b) INFN, Laboratori Nationali di Legnaro, Viale dell' Universita 2, 35020 Legnaro (Padova), Italy

c) GANIL, IN2P3-CNRS and DSM-CEA, BP 55027, F-14076 Caen Cedex5, France

d) Institut de Physique Nucleaire, IN2P3-CNRS, F-91406 Orsay Cedex, France

\begin{abstract}
The capacity of uranium carbide target materials of different structure and density for production of neutron-rich and heavy neutron-deficient nuclides have been investigated. The yields of $\mathrm{Cs}$ and $\mathrm{Fr}$ produced by a $1 \mathrm{GeV}$ proton beam of the PNPI synchrocyclotron and release properties of different targets have been measured. The comparison of the yields and release efficiencies of Cs and $\mathrm{Fr}$ produced from a high density UC target material and from low density $\mathrm{UC}_{\mathrm{x}}$ prepared by the ISOLDE method at IRIS in the collaboration with PARRNe group from Orsay are presented. The yields from ISOLDE original target are presented for comparison as well.
\end{abstract}

PACS. 25.85.-w; 28.60.+S; 29.25-t; 29.25.Rm

\section{Introduction}

This work is a continuation of series of off-line and on-line investigations of different uranium carbide target materials [1,2] with a goal to develop a massive (up to $1 \mathrm{~kg}$ ) uranium carbide target prototype for the third generation ISOL facilities [3,4], where two step reaction will be used to produce intense neutron-rich nuclear beams.

For more than thirty years $\mathrm{UC}_{\mathrm{x}}$ targets $[5,6]$ have been explored for production of a large set of neutron-rich and neutron deficient heavy nuclei. The main direction of uranium carbide target development was the creation of the target material with a high dispersivity and porosity in order to decrease the release time of the produced by different reactions in the target material species which is mainly determined by the diffusion and effusion processes. On the other hand for a more efficient use of a neutron beam after the primary beam converter the density of the target material containing uranium should be as high as possible. Additionally, the target material with a higher density has a higher thermal conductivity that would allow dissipating a higher power introduced in to the target by the primary beam, when the target is used for exotic nuclei production in the direct reaction. Hence, for more effective production of short-lived isotopes of the element chosen for investigation a special selection of a metal carbide target material is required, concerning not only the target material structure, but also its density. At the IRIS (Investigation of Radioactive Ions at Synchrocyclotron) facility in Gatchina in the collaboration PNPI-LNL-GANIL-Orsay uranium carbide target materials of different structure and density have been tested. The yields and release characteristics are reported here to compare properties of explored tested target materials. The general goal of the work is to find out whether high density $\left(11-13 \mathrm{~g} / \mathrm{cm}^{3}\right)$ uranium carbide can be competitive with presently used low density $\mathrm{UC}_{\mathrm{x}}$ targets in the production of short-lived nuclei far from stability. 


\section{Experiment}

A schematic view of the target unit used for different uranium carbide target material tests is shown in fig. 1 a) and fig. $1 \mathrm{~b}$ ). As a first step in that comparison the isotopes of alkali elements Rb, $\mathrm{Cs}$ and Fr have been selected for the yield and release efficiency measurements. The main reason of such a choice was a high efficiency of that specimen ionization (theoretically up to $100 \%$ ) on the hot inner surface of the target-ion source unit, allowing to obtain short-lived nuclei further from stability. This is very important for the target material comparison.

The second important point in the selection of alkalis is that the target construction can be very simple, including only the tungsten target cavity (ionizing target) [7], as the ionization process happens inside the target container itself. It allows to avoid some uncertainties, concerning possible temperature difference of the tested units, as in that case only one parameter (the target container temperature) should be controlled in the course of the experiment.

Three kinds of the target materials were studied: a high density UC rod (fig.1a); a high density UC powder target material prepared by the method of the powder metallurgy; a low density $\mathrm{UC}_{\mathrm{x}}$ target prepared by the ISOLDE method at IRIS in the collaboration PARRNe - IRIS groups (fig.1b). High density rod (HDR) target had the following characteristics: uranium density rod of 11 $\mathrm{g} / \mathrm{cm}^{3}$, the target thickness was $6.7 \mathrm{~g} / \mathrm{cm}^{2}$, about $6 \mathrm{~mm}$ length and $11 \mathrm{~mm}$ in diameter. Low density target $(\mathrm{LDT})$ : uranium density $=2.3 \mathrm{~g} / \mathrm{cm}^{3}$, target thickness $=2.8 \mathrm{~g} / \mathrm{cm}^{2}$, target length was about 12 $\mathrm{mm}$, target diameter $=11 \mathrm{~mm} ; 8$ pellets, each of (1.2-1.6) $\mathrm{mm}$ thickness with the grain size about 20 $\mu \mathrm{m}$. That target material has been prepared by Orsay - PNPI collaborating group at the IRIS facility, using the ISOLDE technology, a similar graphite powder brought by Orsay group and uranium dioxide from Russian producers. High density powder (HDP) target: uranium density $=12$ $\mathrm{g} / \mathrm{cm}^{3}$, target thickness $=6.3 \mathrm{~g} / \mathrm{cm}^{2}$, target length $=5.25 \mathrm{~mm}$, target diameter $=11.2 \mathrm{~mm} ; 3$ pellets, each of (1.6-1.9) $\mathrm{mm}$ thickness, grain size about $20 \mu \mathrm{m}$. Targets were operated in the temperature range of $(1900-2200)^{\circ} \mathrm{C}$. The investigated target materials were maintained in the target containers of the same dimensions and geometry. More detailed description of the used target construction can be found in $[1,8]$. The uranium fission reaction was produced by a $1 \mathrm{GeV}$ protons with the intensity of 50-70 nA. Mass-separated isotope beams were collected on a tape station and transported to $\gamma, \beta$, or $\alpha$ detector to be identified by the characteristic radioactivity. Detailed description of $\gamma$-ray measurement and yield determination can be found in [8]. The yields of Cs isotopes were determined by means of $\beta$-counter as well. The determination of Fr isotope yields were conducted measuring of $\gamma$-rays and $\alpha$-particles emitted by the measured nuclides. To get the consistent yield data obtained by $\alpha$ and $\gamma$ measurements the $\alpha$-yield data were normalized to the yields obtained by $\gamma$-measurement, taking normalizing coefficient as the yield ratio for isotopes measured both by $\gamma$ and $\alpha$ method.

\section{Experimental results and discussion}

\subsection{Yield comparison}

For the investigated target materials the yields $\mathrm{Cs}$ and Fr produced under similar temperature conditions $(\mathrm{T}=2100)^{\circ} \mathrm{C}$ have been determined. All presented yield values were normalized to a target thickness of $1 \mathrm{~g} / \mathrm{cm} 2$ and $0.1 \mu \mathrm{A}$ proton beam current. The error of the temperature measurement for different targets can reach $50{ }^{\circ} \mathrm{C}$. The experimental errors of the obtained yield values are in the limits of $30 \%$ for isotopes not far from stability and of $80 \%$ for short-lived neutron-rich isotopes. Cs yields from both investigated targets are plotted together with the ISOLDE yields $[6,9]$ obtained for standard target at the PS-Booster and for graphite cloth target at SC. As one can see from fig. 2, there is a good agreement of the yields from similar target materials: 
ISOLDE and PARRNe-IRIS. The yields of short-lived Cs isotopes from a HDR target are 1.5-2 times lower that demonstrates its lower release efficiency than ISOLDE or PARRNe - IRIS target. It is expectable result, as the grain size of HDR target is 10 times larger, therefore the diffusion process should be much slower. The yields measured for $\mathrm{Rb}$ isotopes which are not presented here confirm the results obtained for Cs. The Cs yield trend from lately developed HDP pellet target is similar to the fast PARRNe-IRIS target, but the efficiency is about 20 times less. It can be explained by a strong carbonization of the inner surface of the target container that leads to the ionization efficiency decrease, as the HDP target was normalized much longer time than other targets. The calculated yields based on the experimental cross-sections [10] are also presented to demonstrate in target production rate of the investigated isotopes. The enhancement of the yield of ${ }^{139} \mathrm{Cs}$ from the HDR target respectively to the calculated one can be explained by the decay of the mother ${ }^{139} \mathrm{Xe}$ nuclei which have the production cross-section comparable to the Cs daughter nucleus. It worth stressing that the yield excess respectively to the calculated yield values was measured only for Cs isotopes produced from HDR target. It can indicate that in HDR target the Xe precursors may have longer release times than their daughter Cs isotopes. That assumption requires a special verification.

It should be specially pointed out the extraordinary high yields of short-lived $\mathrm{Rb}$ and $\mathrm{Cs}$ obtained from ISOLDE graphite cloth $\mathrm{UC}_{\mathrm{x}}$ target at the SC [9]. Unfortunately these high yield data were not reproduced at the PS-Booster, where presently ISOLDE standard powder-pellet $\mathrm{UC}_{\mathrm{x}}$ target is used.

In fig. 3 the yields of Fr isotopes from a HDR and HDP targets are presented. Fr isotopes have not been measured from PARRNE-IRIS target. The yields of short-lived ${ }^{114} \mathrm{Fr}\left(\mathrm{T}_{1 / 2}=5 \mathrm{~ms}\right),{ }^{118} \mathrm{Fr}$ $\left(\mathrm{T}_{1 / 2}=1 \mathrm{~ms}\right)$ and ${ }^{119} \mathrm{Fr}\left(\mathrm{T}_{1 / 2}=21 \mathrm{~ms}\right)$ were measured by detection their characteristic $\alpha$-decays. The yields of isotopes with longer half-lives were determined using both $\alpha$-particles and $\gamma$-rays. The Fr isotope yields obtained at ISOLDE from $\mathrm{UC}_{\mathrm{x}}$ graphite-cloth target are also shown in the graph. As one can see, the normalized yields of long-lived Fr isotopes are higher from ISOLDE graphite-cloth target than from HDR and HDP targets. The lower HDR and HDP target efficiency, which is the product of ionization and release efficiencies may be explained by lower value of the ionization efficiency of the ionizing targets used for experiments, where the ionization process takes place on the strongly carbonized inner surface of the target container. But, at the same time, the normalized yields of short-lived ${ }^{114,118,119} \mathrm{Fr}$ are equal, or even higher than the yields of these short-lived isotopes from ISOLDE target. As there was only one measurement of the Fr yields from a newly developed HDP target, we give here the obtained yield values from that target as preliminary ones.

\section{Comparison of the target release properties}

The comparison of the relative release efficiencies of different targets for produced isotopes has been derived by normalizing the obtained yield values to the yield of a long-lived isotope ${ }^{140} \mathrm{Cs}$ $\left(\mathrm{T}_{1 / 2}=63.7 \mathrm{~s}\right)$, which produced with the efficiency close to unity. In fig. 4 the relative release efficiencies of $\mathrm{Cs}$ isotopes as a function of their half-lives are presented. The trend of the normalized yield values demonstrates that ISOLDE and ISOLDE like target prepared at IRIS in general are slightly faster than HDR target. A rather promising result is that the trend of the relative efficiency curve for the newly developed HDP target coincidences with one of the ISOLDE target.

For comparison of the Fr release efficiencies the measured yields of isotopes with different halflives were divided by the yields of the same isotopes from ISOLDE cloth target and normalized to the yield ratio of ${ }^{112} \mathrm{Fr}$. The obtained ratio values as function of the half-lives are shown in fig. 5. As the Fr isotopes have not been measured from PARRNe target, in fig. 5 only the ratios for HDR and HDP are presented. The result for HDP target is presented as a preliminary one. As one can see from fig. 5, HDR and HDP targets has considerably higher release efficiency for short livedisotopes of ${ }^{114} \mathrm{Fr}\left(\mathrm{T}_{1 / 2}=5 \mathrm{~ms}\right),{ }^{118} \mathrm{Fr}\left(\mathrm{T}_{1 / 2}=1 \mathrm{~ms}\right)$ and ${ }^{119} \mathrm{Fr}\left(\mathrm{T}_{1 / 2}=21 \mathrm{~ms}\right)$. It can be explained by the fact that the sticking and flight time of produced species in the IRIS tested target prototypes is about six time less than in ISOLDE target due to the ratio of the volumes of the target containers. To 
confirm or reject that assumption some additional on-line tests on the short-lived Fr isotope production from a HDP target will be carried out at IRIS.

\section{Conclusion}

The yields of on-line mass-separated Cs and Fr isotopes have been studied, comparing by IRIS prepared a high density UC targets and a low density $\mathrm{UC}_{\mathrm{x}}$ target prepared by ISOLDE method. According the measured yield trends, the release efficiency of ISOLDE like target for short-lived Cs isotopes is 1.5-2 times higher than a HDR (high density rod) uranium carbide target which was studied at IRIS initially. It was rather encouraging result, as the grain size of HDR UC target was ten times larger than ISOLDE like target.

It should be pointed out that the on-line measured production efficiency and release properties of ISOLDE like target specially prepared at IRIS for that comparison tests coincidence with the ISOLDE original target in the limits of the experimental errors $(\leq 40 \%)$.

To compare the target materials having different densities but a similar dispersivity a new developed, high density pill (HDP) UC target with the grain size of $20 \mu \mathrm{m}$ have been on-line investigated. According to the measured Cs yield trends, the release efficiency of a new developed HDP target coincidences with the ISOLDE like target in the limits of the measurement errors.

The comparison of the yields of short-lived Fr isotopes from HDR and HDP targets with the yields from the ISOLDE graphite-cloth target has demonstrated a rather unexpected result: the normalized yields of extremely short-lived Fr isotopes from high density targets are equal or even higher than from graphite-cloth target. The following step in the HDP uranium carbide target development is the test of a thick $\left(95 \mathrm{~g} / \mathrm{cm}^{2}\right) \mathrm{UC}$ target. The main goal of the thick target development is to convert the high values of normalized yield obtained for small target prototypes to the high values of the absolute yields form a big mass target.

\section{Acknowledgements}

This work was supported by the EC under the FP6 "Research Infrastructure Action- Structuring the Europeasn Research Area" EURISOL DS contract No. 515768 RIDS referring to LNL, GANIL and Orsay and by funding in the frames of ISTC Project No. 2965 referring to PNPI. The EC is not liable for any use that may be made of the information contained herein. 


\section{References}

1. A. Andrighetto, O. Bajeat, A.E. Barzakh, S. Essabaa, D.V. Fedorov, A.M. Ionan, V.S. Ivanov, R. Leroy, G. Lhersonneau, K.A. Mezilev, F.V. Moroz, S.Yu. Orlov, V.N. Panteleev, L. Stroe, L.B. Tecchio, A. Villari, Yu.M. Volkov, and X.F. Wang, Eur. Phys. J. A 23, 257, (2005).

2. V.N. Panteleev, A.E. Barzakh, D.V. Fedorov, A.M. Ionan, V.S. Ivanov, K.A. Mezilev, F.V. Moroz, S.Yu. Orlov, Yu.M. Volkov, A. Andrighetto, E. Del Piero, G. Lhersonneau, L. Stroe, V. Rizzi, L.B. Tecchio, M. Dubois, G. Gaubert, P. Jardin, N. Lecesne, R. Leroy, J.Y. Pacquet,M.G. Saint Laurent, A.C.C. Villari, O. Bajeat, S. Essabaa, C. Lau,M. Menna, Nucl. Instrum. Methods B 240, 888 (2005).

3. A. Bracco, A. Pisent (Eds.) SPES: Technical Design for an Advanced Exotic Ion Beam Facility at LNL, LNL-INFN (Rep.) 181/02 (2002).

4. A. C. C. Villari, in: The SPIRAL2 project at GANIL, AIP Conference Proceedings, vol. 704, p. 234 (2004).

5. H. L. Ravn, Nucl. Instrum. Methods B 70, 107, (1992).

6. A. M. Evensen, R. Catherall, P. Drumm, P. Van Duppen, O.C. Jonsson, E. Kugler, J. Lettry, O. Tengblad, V. Tikhonov, H.L. Ravn, ISOLDE Collaboration, Nucl. Instrum. Methods B 126, 107 (1997).

7. V. N. Panteleev A.E. Barzakh, D.V. Fedorov, A.M. Ionan, K.A. Mezilev, F.V. Moroz, S.Yu. Orlov, Yu.M. Volkov, A. Andrighetto, G. Lhersonneau, V. Rizzi, L.B. Tecchio, M. Dubois, G. Gaubert, P. Jardin, N. Lecesne, R. Leroy, J.Y. Pacquet, M.G. Saint Laurent, A.C.C. Villari, O. Bajeat, S. Essabaa, C. Lau, M. Menna,et al., Rev. Sci. Instrum. 77, 03A705 (2006).

8. A. Andrighetto, A.E. Barzakh, D.V. Fedorov, V.S. Ivanov, G. Lhersonneau, F.V. Moroz, S.Yu. Orlov, V.N. Panteleev, M.D. Seliverstov, I.M. Strachnov, L. Stroe, L.B. Tecchio,a, Yu.M. Volkov, and X.F. Wang, Eur. Phys. J. A 19, 341 (2004).

9. U. Köster, Doctoral Thesis, Dept. Of Physicsof the Munich Technical University (2000).

10. M. Bernas, P. Ambrusteret, J. Benlliure, A. Boudard, E. Casarejos, S. Czajkowski, T. Enqvist, R. Legrain, S.Lelay, B. Mustapha, P. Napolitani, J. Pereire, F. Rejmund, M.-V. Ricciardi, K.-H. Schmidt, C. Stephan, J. Taieb, L. Tassan-Got, C. Volant, Nucl Phys A 725, 213 (2003). 
Fig. 1. Schematic drawings of a) high and low density pellet uranium carbide target; b) high density rod (HDR) uranium carbide target.

Fig. 2. The yields of $\mathrm{Cs}$ isotopes released from a high density rod, high density and low density pellet targets at $2100 \mathrm{C}^{\circ}$. The yields from ISOLDE $\mathrm{UC}_{\mathrm{x}}$ pellet target are presented. The yields calculated on the basis of measured cross-section are shown as well.

Fig. 3. The yields of Fr isotopes from the HDR, HDP and ISOLDE graphite cloth targets.

Fig. 4. Comparison of the Cs yield trends from HDR, HDP, LDP PARRNe-IRIS and ISOLDE targets. The yields are normalized at the yield point of ${ }^{140} \mathrm{Cs}$ with the half-life $\mathrm{T}_{1 / 2}=63.7 \mathrm{~s}$.

Fig. 5. Yields of Fr isotopes from HDR and HDP targets divided by the yields of the same isotopes from ISOLDE graphite cloth target, plotted as a function of the half-lives. The obtained ratios are normalized at the point ${ }^{112} \mathrm{Fr}$ with the half-life $\mathrm{T}_{1 / 2}=20 \mathrm{~min}$. 


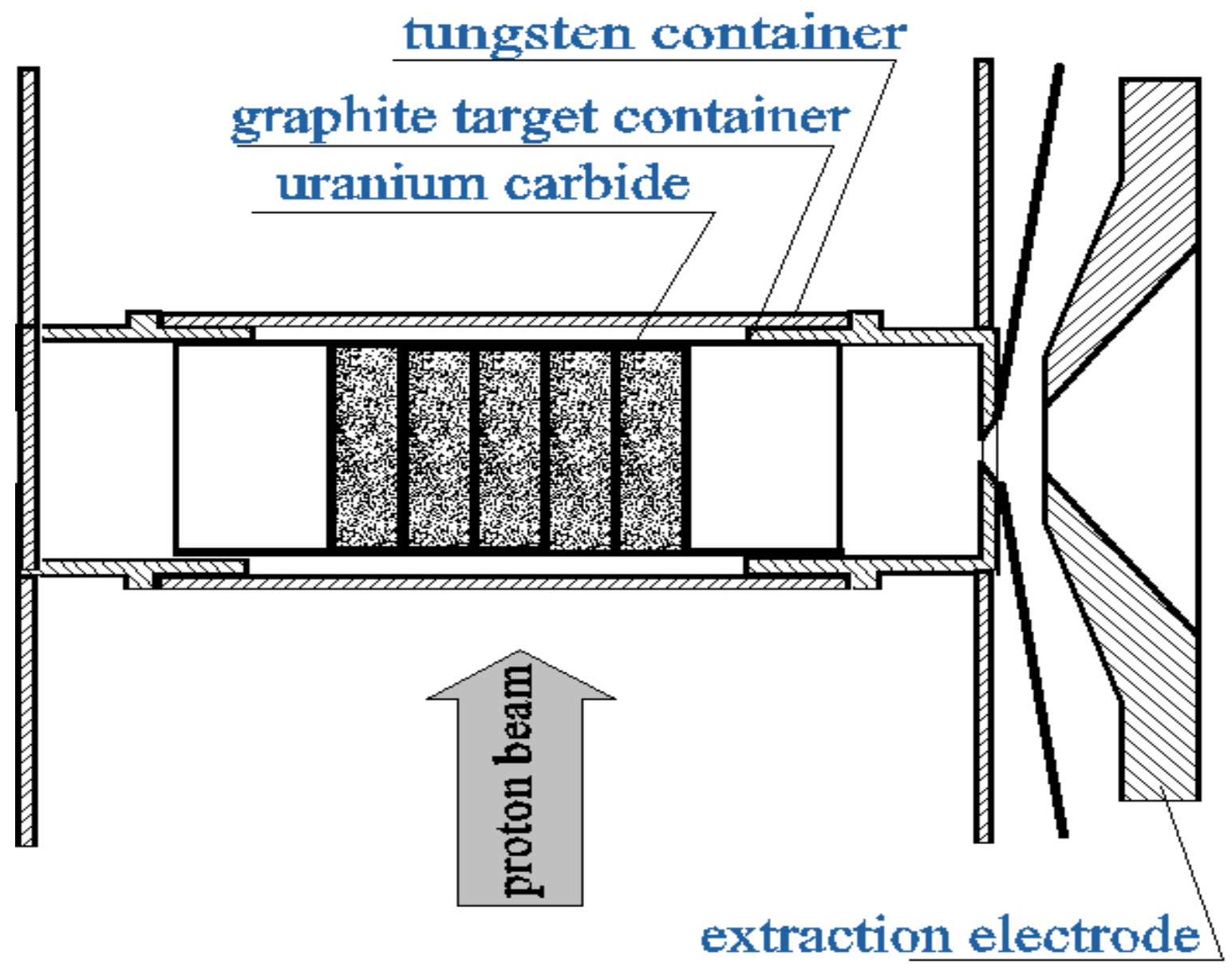

Fig. 1 a) 


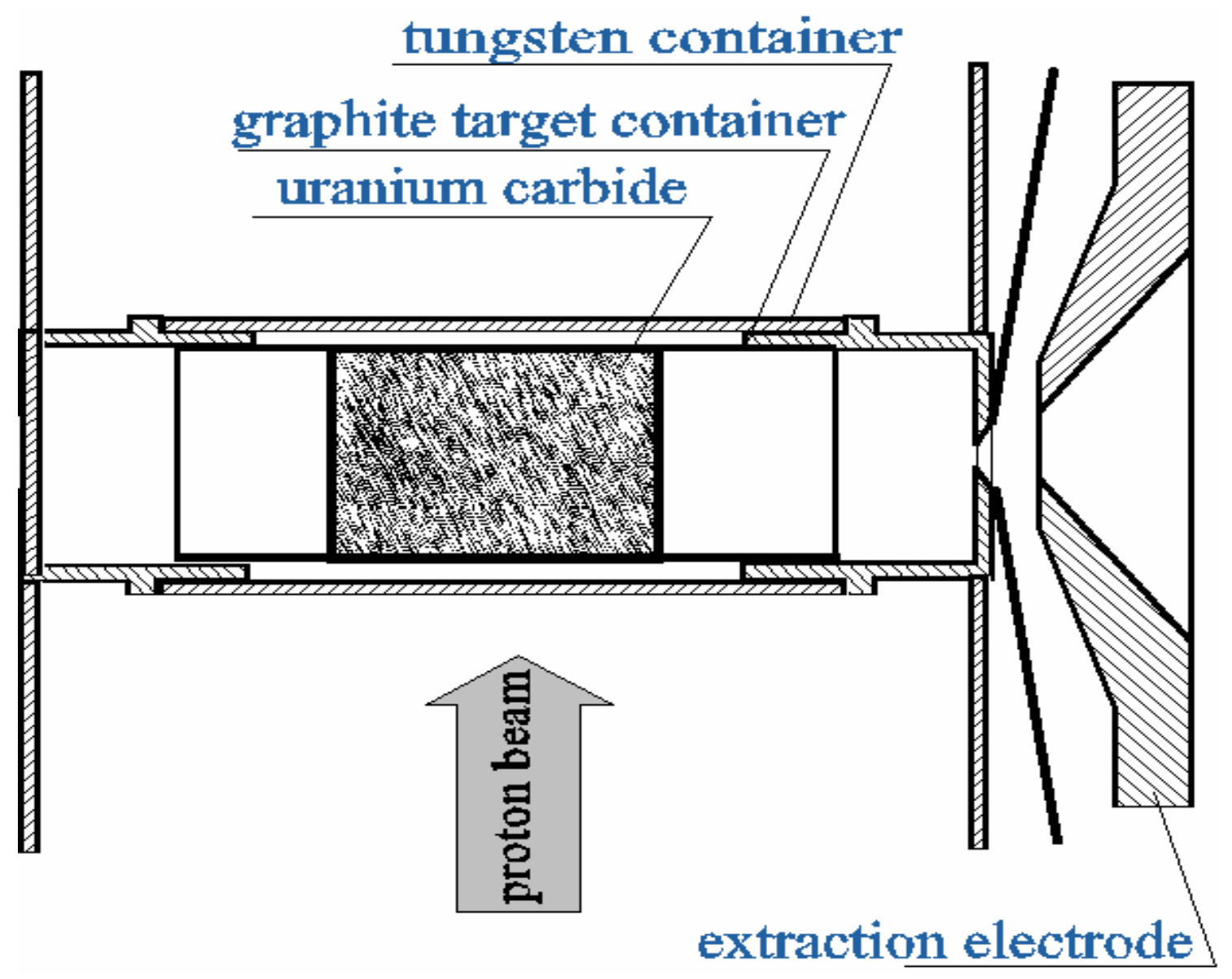

Fig. 1 b) 


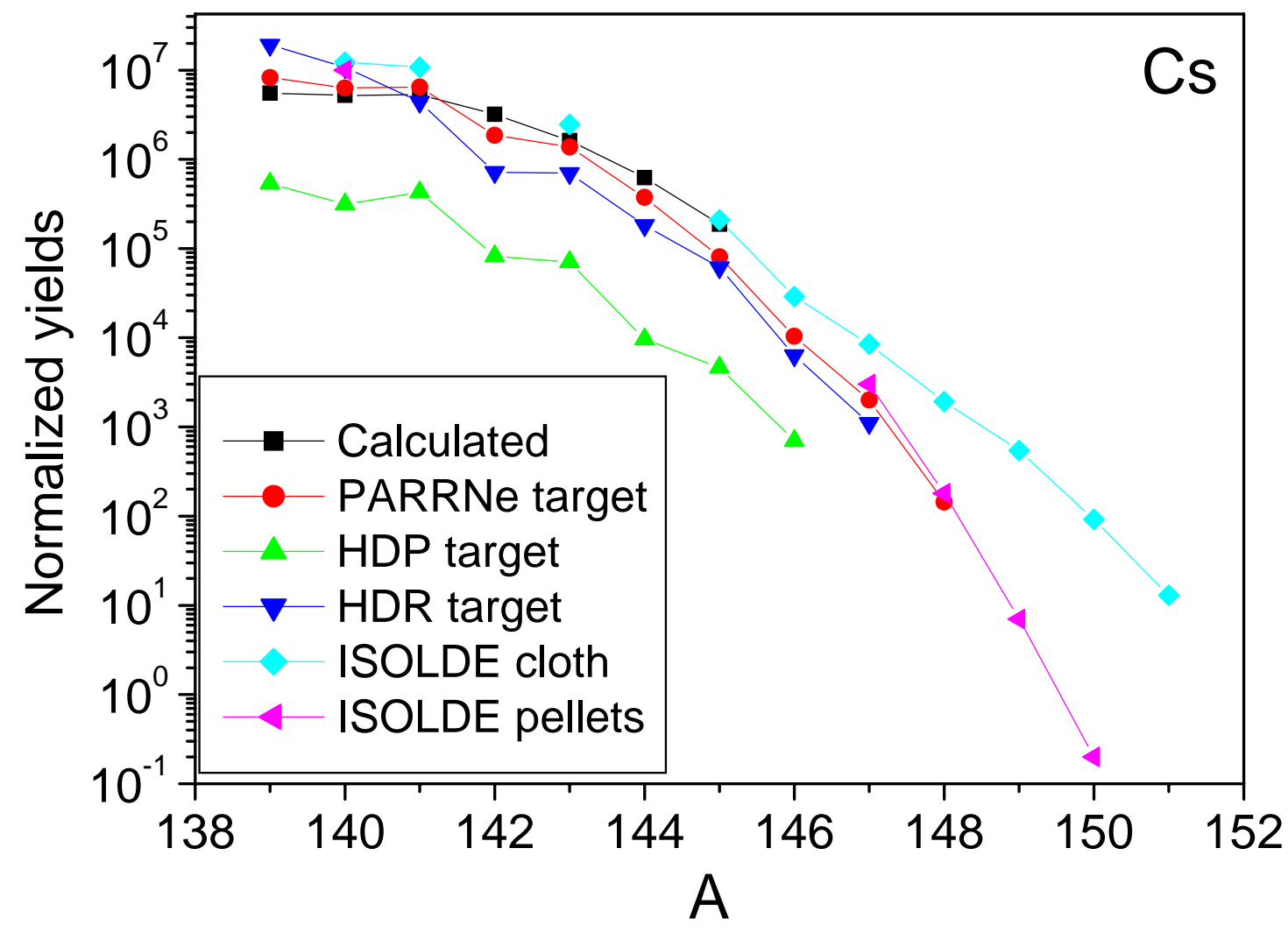

Fig. 2 


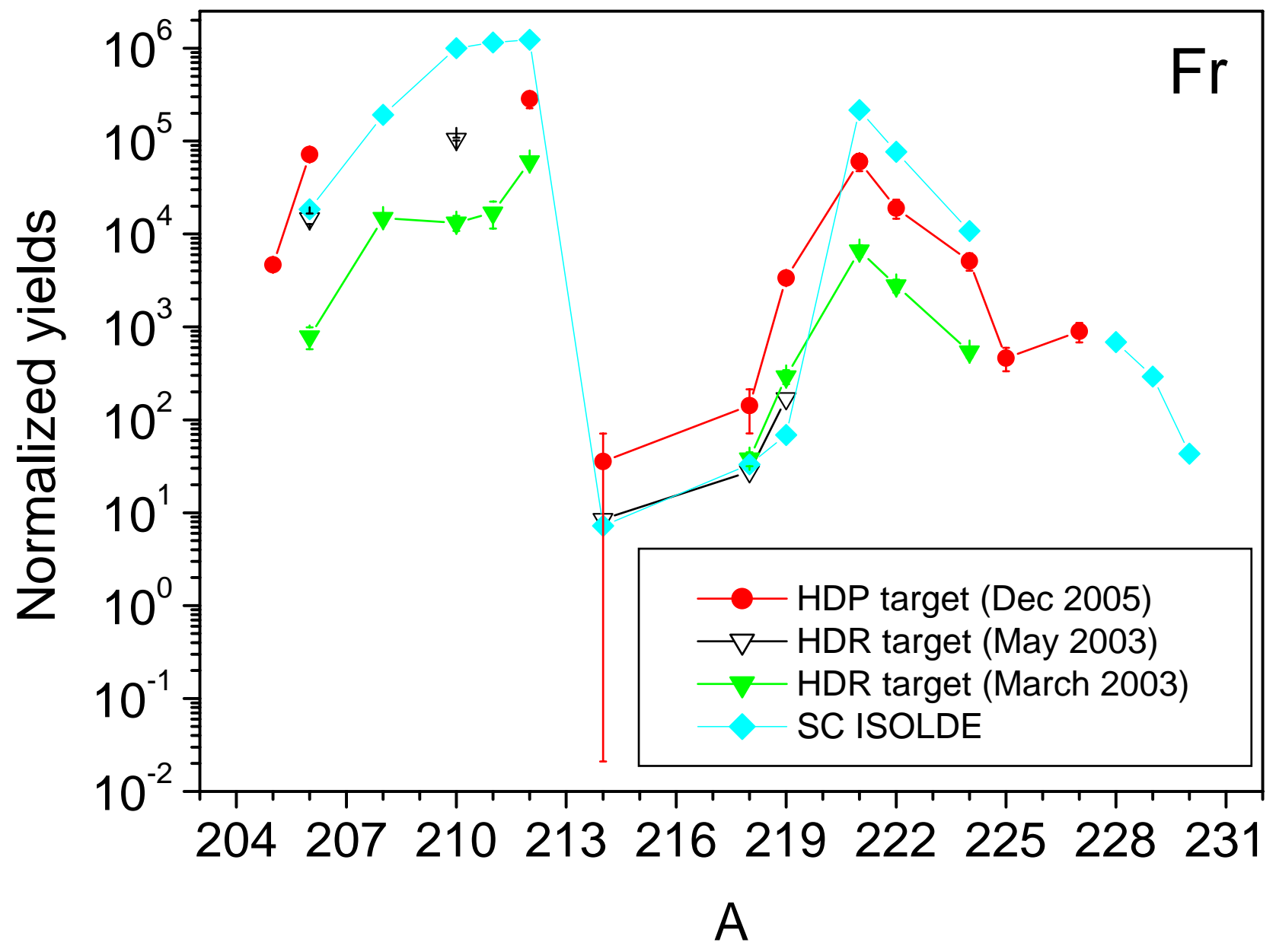

Fig.3 


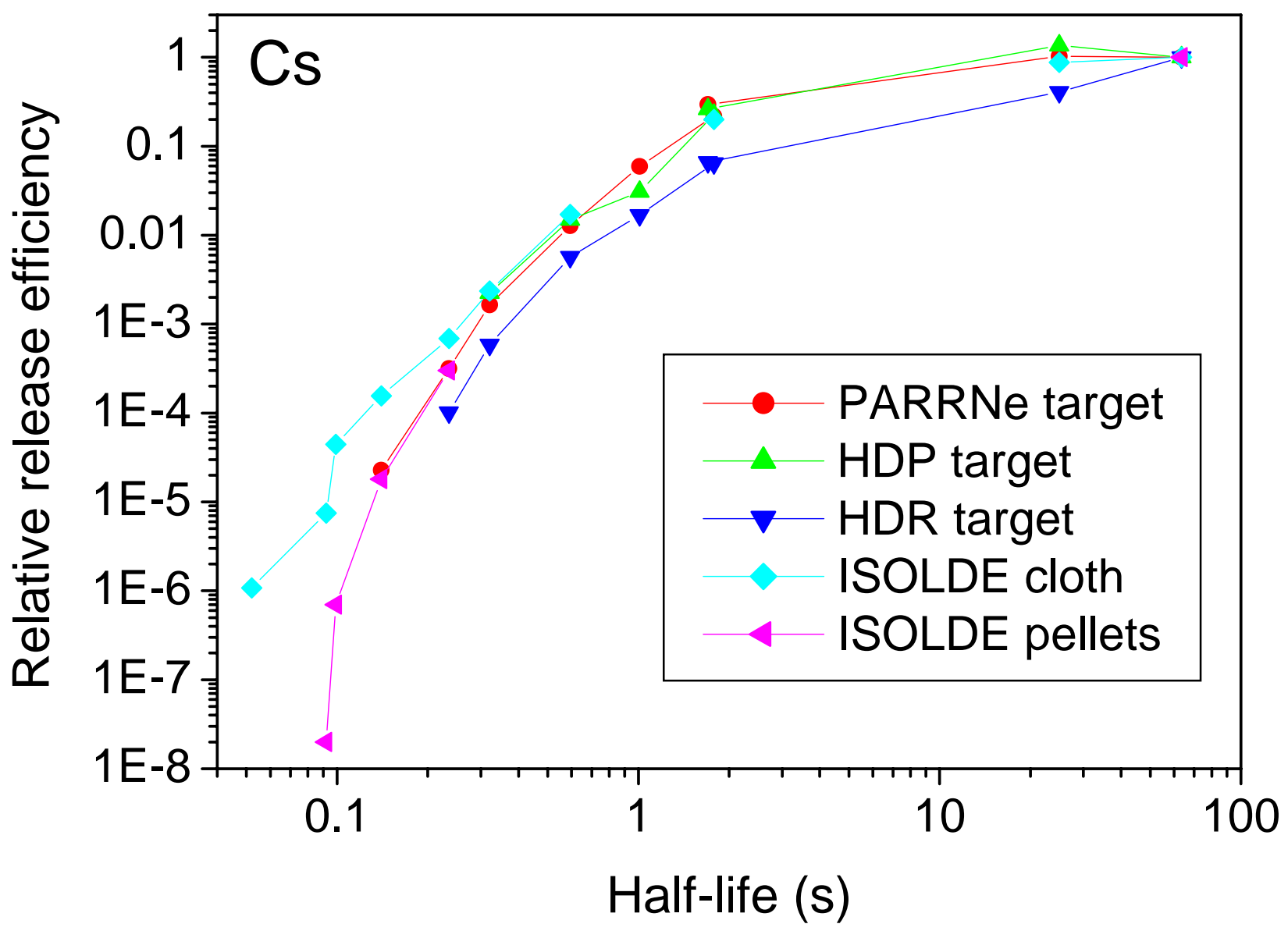

Fig. 4 


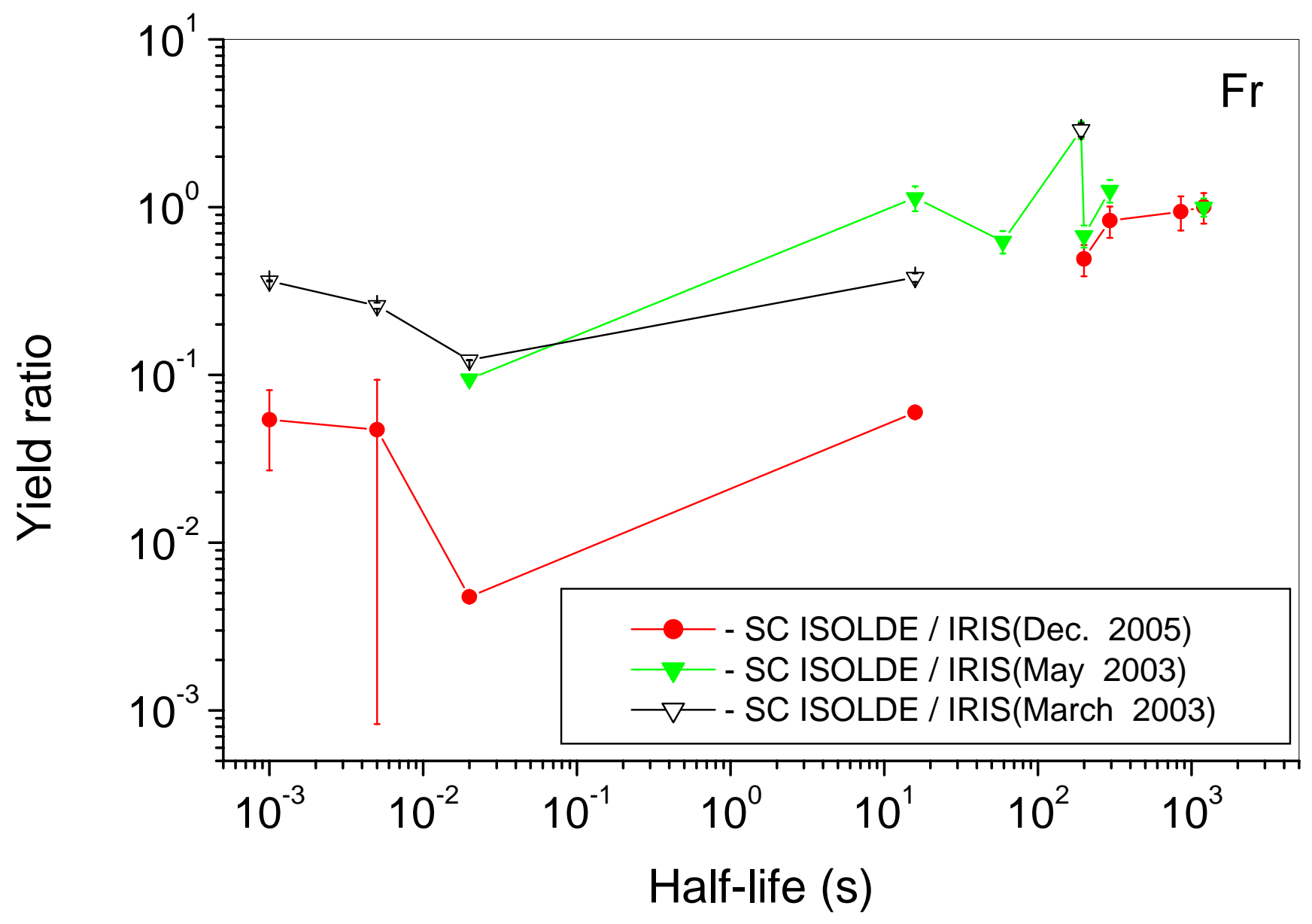

Fig. 5 